\title{
Studies of polymer microring lasers subject to uniaxial stress
}

A. Tulek, and Z. V. Vardeny

Citation: Appl. Phys. Lett. 91, 121102 (2007); doi: 10.1063/1.2785955

View online: http://dx.doi.org/10.1063/1.2785955

View Table of Contents: http://aip.scitation.org/toc/apl/91/12

Published by the American Institute of Physics

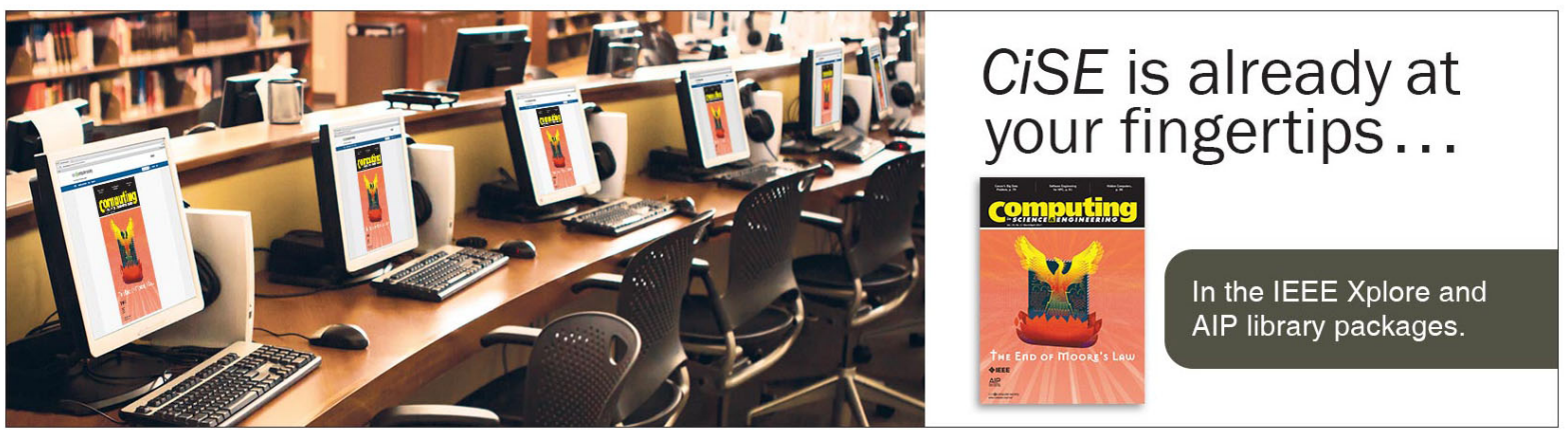




\title{
Studies of polymer microring lasers subject to uniaxial stress
}

\author{
A. Tulek ${ }^{\mathrm{a})}$ and Z. V. Vardeny ${ }^{\mathrm{b})}$ \\ Department of Physics, University of Utah, Salt Lake City, Utah 84112, USA
}

(Received 26 June 2007; accepted 28 August 2007; published online 17 September 2007)

\begin{abstract}
The emission spectra of microring lasers fabricated from $\pi$-conjugated polymer films casted on nylon microfibers with diameters in the range of 35-90 $\mu \mathrm{m}$ were studied upon application of uniaxial stress with strain up to $\sim 12 \%$. The laser emission spectra substantially change with the applied stress, showing enhanced sensitivity to stress over changes induced in the fiber diameter alone. This is explained as due to the induced change in the polymer refractive index spectrum upon stress, causing an unexpected increase in the refractive index dispersion and, consequently, also in the effective refraction index for lasing at emission wavelengths. (C) 2007 American Institute of Physics. [DOI: 10.1063/1.2785955]
\end{abstract}

In a microring cavity light is confined by total internal reflection at the interface between the active medium and air due to the discontinuity in the refractive index $n$ that changes at the interface from high ( $n>1$ in the active medium) to low ( $n \cong 1$ in air). Such a morphology-related light confinement also provides the positive feedback needed for stimulated emission amplification that is essential for laser action. Following the first demonstration of Weber and Ulrich ${ }^{1}$ many studies of characterizing microring lasers have been completed, including the investigation of spectral characteristics, ${ }^{2}$ multimode dynamics, ${ }^{3}$ and relative intensity noise due to nonlinear mode competition. ${ }^{4}$ Enhanced directionality $^{5,6}$ and tunability ${ }^{7}$ were also obtained using microring lasers coupled with a waveguiding element outside the microcavity. Recently, electrically pumped inorganic microring lasers were reported using InGaAsP-InP as a gain medium. ${ }^{5,8}$ For fabrication of such microlasers, soft lithography ${ }^{6}$ and nanoimprint ${ }^{9}$ techniques were utilized as well as conventional lithographic methods. In addition to laser studies in inorganic gain media, many organic microring lasers have also been investigated during the last decade using a variety of gain media including high efficiency dyes $^{10,11}$ and a number of $\pi$-conjugated polymers. ${ }^{12-15}$

One of the distinct characteristic properties of organic gain media is that they are mechanically flexible. This property may be used in changing the microcavity shape by applying moderate external forces such as stress, while maintaining the laser action. Because the microlaser intermode frequency separation directly depends on the effective refractive index $n_{\mathrm{eff}}$ of the gain medium, it should be possible to monitor the microcavity deformation by studying the laser emission spectrum of organic microcavities, from which the change in $n_{\text {eff }}$ induced by the external forces may be readily obtained. In this report we studied the deformation of $\pi$-conjugated polymer microring lasers by applying uniaxial stress. We report a large decrease in $n_{\text {eff }}$ with stress, which leads to superior sensitivity of $\pi$-conjugated polymer gain media of registering stress. We show that the superior sensitivity in monitoring $n_{\text {eff }}$ in polymer laser comes from the dependence on the dispersion in $n$ via the relation, ${ }^{16}$

\footnotetext{
${ }^{a)}$ Present address: Department of Physics, Bilkent University, 06800 Bilkent, Ankara, Turkey.

${ }^{\text {b) }}$ Author to whom correspondence should be addressed. Electronic mail: val@physics.utah.edu
}

$$
n_{\mathrm{eff}}=n-\lambda \frac{d n}{d \lambda},
$$

where $\lambda d n / d \lambda$ is the first order dispersion term and $\lambda$ is the lasing wavelength. We demonstrate that the dispersion term in Eq. (1) changes much more than $n$ upon stretching the polymer film, thus providing the superior sensitivity of organic laser for detecting stress.

The polymer microring lasers were fabricated by drop casting a polymer solution onto nylon fibers of cylindrical shape with diameter in the range of $35-90 \mu \mathrm{m}$. The $\pi$-conjugated polymer used in this work was a soluble derivative of poly(phenylene vinylene) (PPV), namely, dioctyloxy PPV (DOO-PPV), which is known to be an excellent laser gain medium. ${ }^{14}$ Usually a polymer layer of $\sim 1 \mu \mathrm{m}$ thick is deposited onto the fiber when the drop-casted film dries. The coated fiber was then mounted onto a specially engineered stretching unit that was designed to apply a predetermined force on the fiber [see inset of Fig. 2(a)], in which the fiber length $L$ was increased by increments of $0.5 \mathrm{~mm}$. The new fiber length $L_{f}$ and diameter $D_{f}$ were monitored in situ by a microscope. The polymer microring was optically pumped using a pulsed Nd:yttrium-aluminumgarnet laser amplifier system operating at $532 \mathrm{~nm}$ with a pulse duration of $100 \mathrm{ps}$ at $870 \mathrm{~Hz}$ repetition rate, where the excitation beam of various intensities was focused onto the organic microring cavity using a cylindrical lens. The emitted light from the microring laser was collected using an optical fiber of $1 \mathrm{~mm}$ diameter placed at a distance of less than $50 \mu \mathrm{m}$, and sent to a triple spectrometer, where a charged-coupled device camera recorded the emission intensity in units of photon counts. The overall spectral resolution of the measurement apparatus was $\sim 0.1 \mathrm{~nm}$. Also in order to avoid photo-oxidation of the DOO-PPV polymer, all measurements were performed in a chamber under dynamical vacuum of $\sim 10^{-3}$ torr.

Figure 1(a) shows the emission spectrum of a $37 \mu \mathrm{m}$ diameter polymer microring above the laser threshold. The characteristic bi-linear dependence of the emission intensity versus the excitation energy is displayed in Fig. 1(a) (inset) showing the existence of a laser threshold at $\sim 25 \mathrm{~nJ} /$ pulse; this translates into a laser $Q$ value of about $5 \times 10^{3}$, similar to those obtained in previous studies. ${ }^{13,14}$ For analyzing the laser spectrum we recall that the spectral spacing $\Delta \lambda$ between adjacent laser modes is given by the following relation: ${ }^{17}$ 
(a)

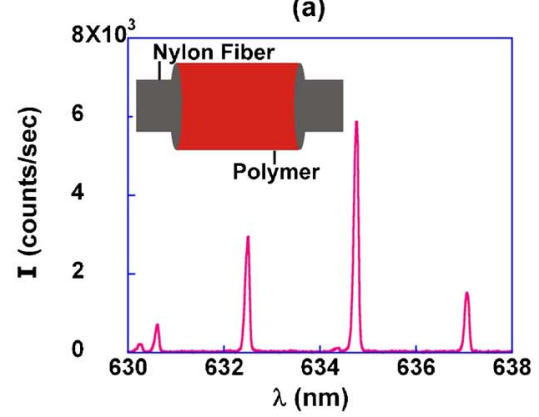

(b)

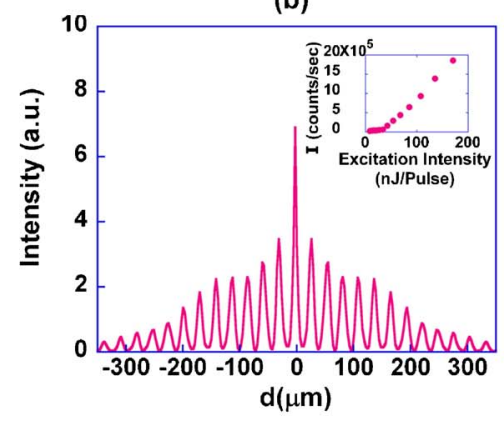

(c)

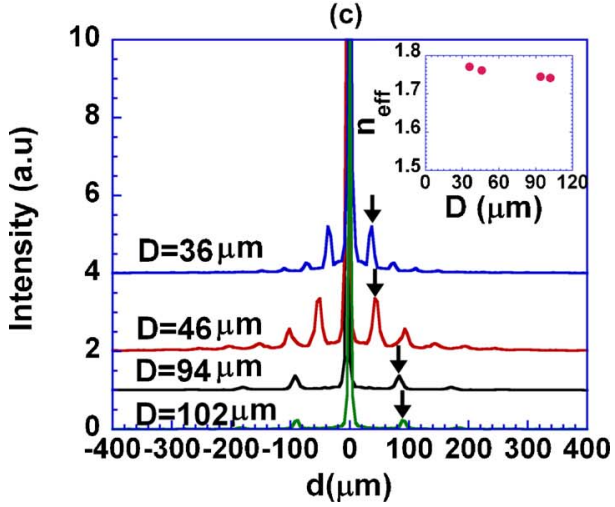

FIG. 1. (Color online) (a) Emission spectrum of a microring laser (see inset) fabricated from DOO-PPV polymer on a nylon microfiber $\sim 37 \mu \mathrm{m}$ in diameter measured above the laser threshold. (b) Power Fourier transform (PFT) spectrum of the emission spectrum in (a). The inset shows the emission intensity vs. excitation intensity, with laser threshold at $\sim 25 \mathrm{~nJ} / \mathrm{pulse}$. (c) PFT of polymer microring laser emission spectra having various diameters as indicated. The inset shows the obtained $n_{\text {eff }}$ for the gain medium using Eq. (3).

$$
\Delta \lambda=\lambda^{2} / \pi n_{\mathrm{eff}} D,
$$

where $D$ is the microring diameter. However, it was previously found $^{17}$ that the power Fourier transform (PFT) of the emission spectrum is more useful in monitoring the resonant line spacing because it contains equally spaced, diminishing discrete FT components [Fig. 1(b)] having separation $\Delta d$, given by the relation,

$$
\Delta d=n_{\mathrm{eff}} \mathrm{D} / 2,
$$

where $d$ is the FT "length" parameter. We therefore registered the induced change in the microring $n_{\text {eff }} D$ parameter upon stretching the fiber by studying the laser emission PFT using Eq. (3).

The PFT of laser emission spectra of several polymer microrings having various diameters are shown in Fig. 1(c). It is seen that their FT discrete components shift toward $d$ $=0$, and thus $\Delta d$ indeed decreases at smaller $D$, in agreement with Eq. (3). We calculated $n_{\text {eff }}$ for the microring lasers in Fig. 1(c) using Eq. (3) and found that $n_{\text {eff }} \sim 1.74$ and is independent of $D$ [Fig. 1(b), inset]. This finding is very important for the stretching measurements described below, since the induced change in $n_{\text {eff }}$ that we obtained upon stretching does not come from a change in $D$, but instead reflects changes in the polymer optical constants upon stretching [Eq. (1)].

In Fig. 2(a) we show the change in the emission spectrum of a $37 \mu \mathrm{m}$ diameter microring laser induced upon uniaxial stretch up to $\sim 12 \%$ strain; the corresponding PFT of the emission spectra are shown in Fig. 2(b). Although there is a little change in the laser threshold intensity upon stretching showing that the laser $Q$ value $^{13,14}$ stays put; on the contrary, there is a large change in the laser emission spectrum. To better present the shift of the discrete FT components upon stretching, in Fig. 2(b) we emphasize by arrows the induced change of the fourth harmonic. It is clearly seen that upon stretching the discrete FT components shift toward $d$ $=0$, and thus $\Delta d$ decreases in agreement with the expected decrease in the fiber diameter upon stretching, similar to that shown in Fig. 1(c). However, the change in $\Delta d$ is much larger than that expected from the stress induced change in $D$ when using the induced strain and Poisson ratio to calculate $\Delta D$.

Figure 3(a) shows the change in the laser parameter $n_{\text {eff }} D$ calculated from Fig. 2(b) using Eq. (3), as well as the corresponding change in the fiber diameter $D$ measured by an optical microscope, as a function of the applied longitudinal strain $\Delta L / L_{0}$. It is seen that the induced change in $n_{\mathrm{eff}} D$ is much larger than that of $D$. By comparing the two sets of data, we also calculated the induced change in the laser parameter $n_{\text {eff }}$ upon stretching [Fig. 3(b)]. A large decrease in $n_{\text {eff }}$ is realized upon stretching, which is roughly the same as that of the $\sim 12 \%$ change in $D$ induced upon stretching. The changes in $n_{\text {eff }}$ and $D$ then add up for a total of $\sim 25 \%$ (a)

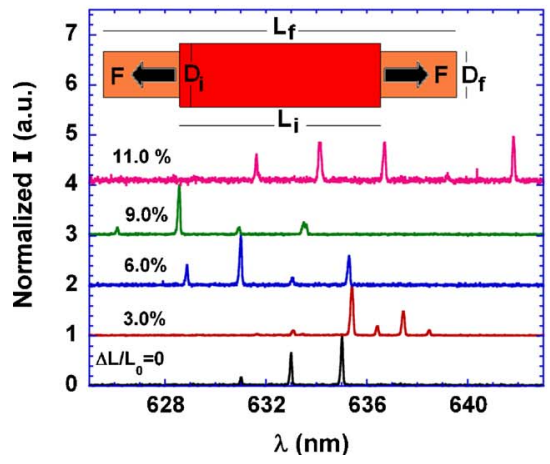

(b)

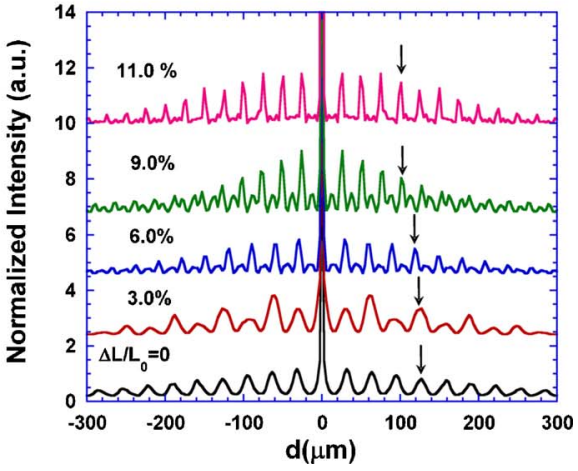

FIG. 2. (Color online) (a) Laser emission spectra and (b) the corresponding PFT of a polymer microring laser subjected to uniaxial stress, with strain up to $12 \%$ as indicated. The arrows in (b) point to the fourth discrete FT harmonics for better showing the decrease in $\Delta d$ induced upon stretching. The inset of (a) shows the stretching direction, the initial $\left(L_{i}\right)$ and final $\left(L_{f}\right)$ lengths, and diameters $D_{i}$ and $D_{f}$, respectively. 


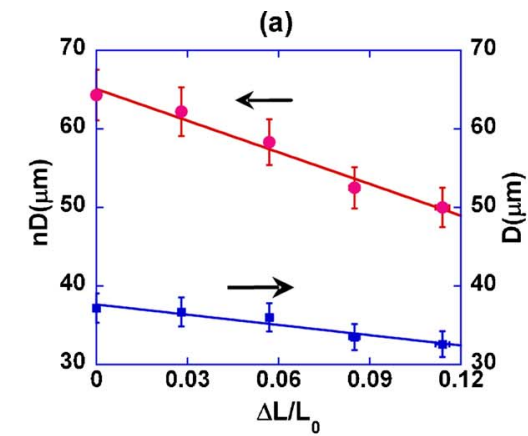

(b)

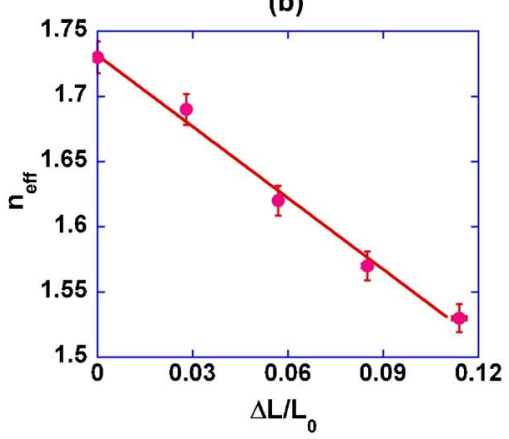

(c)

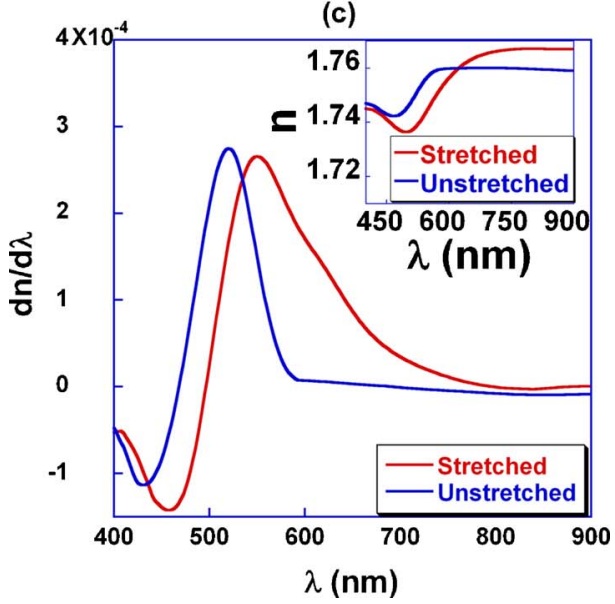

FIG. 3. (Color online) (a) Change in the laser parameter $n_{\mathrm{eff}} D$ (red circles; left scale) induced upon stretching obtained from the analysis of the emission spectra in Fig. 2 using Eq. (3); the corresponding changes in $D$ (blue squares; right scale) measured by an optical microscope are also given for comparison. (b) Calculated change in $n_{\text {eff }}$ induced upon stretching, as obtained from the data in (a). (c) Refraction index dispersion spectrum $d n / d \lambda$ of an unstretched (blue line) and stretched (red line) polymer film on a nylon substrate subjected to strain of $\sim 5 \%$. The inset shows the corresponding change in $n(\lambda)$ spectrum plotted with the same color code.

decrease in the value of the laser parameter $n_{\mathrm{eff}} D$ upon stretching; this represents a superior sensitivity of the polymer lasers in monitoring stress.

In order to understand the nature of such a dramatic change in $n_{\text {eff }}$ induced upon stretching, $d n / d \lambda(\lambda)$ and $n(\lambda)$ spectra were obtained for a stretched $\left(\Delta L / L_{0} \sim 5 \%\right)$ and unstretched polymer film from the optical transmission spectrum $T(\lambda)$ [Fig. 3(c)] for both polarizations parallel and perpendicular to the stretching direction. For these measurements a polymer film of $\sim 0.6 \mu \mathrm{m}$ was coated onto a nylon substrate by spin casting, and the transmission spectrum was measured with polarization parallel and perpendicular to the stretching direction. For obtaining $n(\lambda)$ spectrum the complex refractive index $\boldsymbol{n}=n+i k$ spectrum was calculated from $T(\lambda)$ using an effective medium approxima- tion method. ${ }^{18} n(\lambda)$ become anisotropic upon stretching; we observed very little change in $n(\lambda)$ for polarization perpendicular to the stretching direction. However, for polarization parallel to the stretching direction it is observed [Fig. 3(c)] that $n(\lambda)$ spectrum broadens and redshifts upon stretching and, consequently, the dispersion $d n / d \lambda$ dramatically increases at the laser emission wavelength $\left(\lambda_{0} \sim 635 \mathrm{~nm}\right)$. The induced changes in $n(\lambda)$ upon stretching are probably due to reversible chain reorientation in the stretching direction; for microring lasing, the change in $n(\lambda)$ parallel is more important. $^{13,14}$ In fact, when calculating $n_{\text {eff }}(635 \mathrm{~nm})$ parallel using Eq. (1), where $n=1.73$ and $\lambda d n / d \lambda=0.07$ [Fig. 3 (c)] we obtain $n_{\text {eff }}=1.66$ for the stretched polymer film. Assuming that $\lambda d n / d \lambda$ increases linearly with the applied strain, and taking into account that the measured film was subjected to a strain of $\sim 5 \%$, then we expect a reduction in $n_{\text {eff }}$ of about $0.17(\sim 10 \%)$ for a $12 \%$ strain. This agrees very well with the obtained reduction of $\sim 12 \%$ in $n_{\text {eff }}$ obtained for the stretched polymer microcavity when a $12 \%$ strain is applied [Fig. 3(b)], explaining the sensitivity of the polymer lasers to monitor stress via $n_{\text {eff }}$.

In conclusion, we measured a substantial decrease in the effective refractive index of the gain medium in a microring laser fabricated from a $\pi$-conjugated polymer operating under uniaxial stress. The enhanced sensitivity of $n_{\text {eff }}$ to the applied stress is attributed to the induced change in the polymer refractive index dispersion parallel to the stretching direction upon the application of uniaxial stress, together with the influence of this dispersion on the laser effective refraction index that determines the laser emission spectrum.

We thank Dr. R. Polson for useful discussions and help with the measurements. This work was supported in part by the DOE Grant No. 04-ER 46109, and the NSF DMR Grant No. 05-03172 at the University of Utah.

${ }^{1}$ H. Weber and R. Ulrich, Appl. Phys. Lett. 19, 38 (1971).

${ }^{2}$ B. Corbett, IEEE Photonics Technol. Lett. 10, 3 (1998).

${ }^{3}$ I. Stamataki, S. Mikroulis, A. Kapsalis, and D. Syvridis, IEEE J. Quantum Electron. 42, 1266 (2006).

${ }^{4}$ S. Mikroulis, I. Stamataki, D. Alexandropoulos, M. Hamacher, E. Roditi, and D. Syvridis, IEEE Photonics Technol. Lett. 18, 1895 (2006).

${ }^{5}$ S. Park, S. S. Kim, L. Wang, and S. T. Ho, IEEE J. Quantum Electron. 41, 351 (2005).

${ }^{6}$ Y. Huang, G. T. Paloczi, J. Scheuer, and A. Yariv, Opt. Express 11, 2452 (2003).

${ }^{7}$ A. Kapsalis, I. Stamataki, S. Mikroulis, D. Syvridis, and M. Hamacher, IEEE Photonics Technol. Lett. 18, 261 (2006).

${ }^{8}$ K. Amarnath, R. Grover, S. Kanakaraju, and P. T. Ho, IEEE Photonics Technol. Lett. 17, 2280 (2005).

${ }^{9}$ C. Chao and L. Jay Guo, J. Vac. Sci. Technol. B 20, 2862 (2002).

${ }^{10}$ M. K. Gonokami, R. H. Jordan, A. Dodabalapur, H. E. Katz, M. L. Schilling, R. E. Slusher, and S. Ozawa, Opt. Lett. 20, 2093 (1995).

${ }^{11}$ G. Jordan, T. Kobayashi, and W. J. Blau, J. Opt. Soc. Am. B 24, 808 (2007).

${ }^{12}$ R. C. Polson, G. Levina, and Z. V. Vardeny, Appl. Phys. Lett. 76, 3858 (2000).

${ }^{13}$ S. V. Frolov, Z. V. Vardeny, and K. Yoshino, Appl. Phys. Lett. 72, 1802 (1998).

${ }^{14}$ S. V. Frolov, M. Shkunov, Z. V. Vardeny, and K. Yoshino, Phys. Rev. B 56, R4363 (1997).

${ }^{15}$ Y. Yoshida, Y. Nishihara, A. Fujii, M. Ozaki, K. Yoshino, H. K. Kim, N. S. Baek, and S. K. Choi, J. Appl. Phys. 95, 4193 (2004).

${ }^{16}$ M. Kneissl, M. Teepe, N. Miyashita, N. M. Johnson, G. D. Chern, and R. K. Chang, Appl. Phys. Lett. 84, 2485 (2004).

${ }^{17}$ A. Tulek and Z. V. Vardeny, Appl. Phys. Lett. 90, 161106 (2007).

${ }^{18}$ S. Bosch, J. F. Borrull, N. Leinfellner, and A. Canillas, Surf. Sci. 453, 9 (2000). 